\title{
Comparison of numerical models for prediction of pressure-swirl atomizer internal flow
}

\author{
Milan Maly*1, Jaroslav Slama ${ }^{2}$, Ondrej Cejpek ${ }^{1}$ and Jan Jedelsky ${ }^{1}$ \\ ${ }^{1}$ Brno University of Technology, Technicka 2, 61669 Brno, Czech Republic \\ 2Provyko s.r.o, Vinarska 460, 60300 Brno, Czech Republic \\ *Corresponding author: milan.maly@vutbr.cz
}

\begin{abstract}
Numerical prediction of discharge parameters allows to design a pressure-swirl atomizer in fast and cheap manner, yet it must provide reliable results for a wide range of geometries and operating regimes. Many authors used different numerical setups for similar cases and often concluded opposite suggestions on numerical setup. This paper compares 3D (threedimensional) periodic numerical models used for estimation of the internal flow characteristics of a pressure-swirl atomizer. The computed results are compared with experimental data in terms of spray cone angle, discharge coefficient $\left(C_{D}\right)$, internal air-core dimensions, and velocity profiles.

The internal air-core was visualized by high-speed camera with backlit illumination. Tested conditions covered wide range of the Reynolds numbers within the inlet ports, $R e=1000$, 2000,4000 . The flow was treated as both steady and transient flow. The numerical solver used laminar and several turbulence models, represented by $k-\varepsilon$ and $k-\omega$ models, Reynolds Stress model (RSM) and Large Eddy Simulation (LES). The laminar solver was capable to closely predict the $C_{D}$, air-core dimensions and velocity profiles compared with the experimental results. The LES performed similarly to the laminar solver for low $R e$ and was slightly superior for $R e=4000$. The two-equation models were sensitive to proper solving of the near wall flow and were not accurate for low Re. Surprisingly, the RSM produced worst results.
\end{abstract}

\section{Keywords}

Internal flow, CFD, Laminar, air-core

\section{Introduction}

Pressure - swirl atomizers (PS) play a unique role in many industrial applications including combustion, spray cooling, spray drying, etc. A typical PS atomizer contains tangential entry ports and a swirl chamber with an exit orifice. The liquid is fed via tangential ports into the swirl chamber where it gains high swirl velocity. The swirling liquid is discharged from the exit orifice in the form of a hollow conical liquid sheet which consequently disintegrates into filaments and ligaments. Despite the simple geometry, the internal flow behaviour is complex. The swirling liquid creates a low-pressure zone along a centre line of the swirl chamber where an air-core is presented. The internal vortex behaves as a Rankine vortex since the swirl velocity has its maximum located at the air-core surface which behaves like a virtual solid cylinder [1].

It is well known that the internal flow directly affects the parameters of the discharged liquid sheet, such as its thickness, stability, velocity, and SCA. These parameters consequently determine the sizes of ligaments and droplets. To understand the link between the atomizer performance and its design, the internal flow must be examined. Some authors used the exact analytical solution to predict the discharge parameters. Simple non-viscous treatment proved to be a useful tool for a basic insight into the flow behaviour, but it lacks accuracy for some atomizer geometries. A better agreement can be achieved when the viscous flow is assumed 
$[2,3]$, but still some aspects such as the liquid sheet temporal stability or secondary flow effects, are not resolved.

On the other hand, the numerical simulation has arisen in recent years due to an increase in a computational performance, and many commercial software are available in the market. These software tools for numerical simulations of the flow dynamics could be simply applied for predicting of the internal flow of the PS atomizer. However, many different geometrical, numerical and physical setup combinations can be used for the same atomizer and operating conditions. The CFD simulation of the internal flow ideally suits for final adjustments of the atomizer geometry, since it allows for fine-tuning of the atomizer individual parts and captures the internal flow instabilities.

In the past, many authors performed a CFD simulation of the internal flow of PS atomizer. One of the first numerical studies of the PS atomizer was conducted in 1997 by Yule and Chinn [4]. They used a 2D axisymmetric geometry with a laminar solver and reported a deviation of discharge coefficient, $C_{D}$, from an experimental data to be less than $3 \%$. A similar numerical setup was later used by Amini [2]. Even this simple 2D model yield better agreement with the experimental data than the analytical viscous solution. Note here that the author used the laminar solver even for values of Reynolds number within the inlet port, $R e=122,000$. The port-based $R e$ is defined as $R e=v_{p} d_{p} \rho_{l} / \mu_{l}$, where $v_{p}$ is velocity within the inlet port, $d_{p}$ is the inlet port hydraulic diameter, $\mu_{l}$ is liquid dynamic viscosity and $\rho_{l}$ is liquid density. The complex nature of the internal flow does not allow for a simple conclusion whether the flow is turbulent or laminar, and many authors claim the opposite. A theoretical evaluation of turbulence evolution within the swirl chamber was made by Yule and Chinn $[5,6]$ who suggest that the flow is laminar even for very high $R e$ due to the laminarization effect of the swirl dominant flow itself and also due to very short length scale where the turbulence has no time to develop.

A comparison of Large Eddy Simulation (LES) and laminar models was performed by Madsen et al. [7]. They used a scaled atomizer and operated it in range of $R e=12,000-41,000$. Under these operating regimes, the laminar model had a slightly better agreement to the experimental data than LES. The authors also examined simple turbulence models represented by RNG (renormalization group) and realizable $k-\varepsilon$ models. However, these models were unable to predict the internal air-core. Galbiati et al. [8] compared the LES simulation with RNG $k-\varepsilon$ and RSM (Reynolds stress model). They found an insignificant variation among the used models when compared to the deviation in the results from published empirical correlations. They also noted that the flow field was consistent for LES and $k-\varepsilon$ model, while, surprisingly, the RSM had some discrepancies. Qian [9] found RSM to be superior over the laminar model at $R e=16,000$, while Nouri-Borujerdi [10] found opposite conclusions for even larger $R e$ in range of 18,000-40,000.

Baharanchi et al. [11] examined several schemes to capture the liquid-air interface using a 2D simulation with RNG $k-\varepsilon$ turbulence model. A geo-reconstruct scheme was found to be the most suitable for capturing the air-core. They also discussed the necessity to include surface tension and found that it has an effect only for Weber number smaller than 204. From a practical point of view, it had a negligible effect on the developed flow. However, it affects the flow development process.

\section{Material and Methods}

The experiments were performed at specially designed facility for cold atomizer testing at Brno University of Technology, Czech Republic. A similar experimental setup was also used in our previous study where it is described in greater detail [12]. 


\section{The atomizer design and test bench}

The atomizer geometry was derived from a small-sized atomizer studied in our previous work [13]. Three inlet ports are used to take advantage of the circumferential periodicity of the atomizer. The swirl chamber has a conical converging part to aid manufacturing simplicity. The atomizer is manufactured as ten times scaled copy since the small dimension of the original atomizer does not allow for direct optical measurement. The transparent parts of the atomizer are made from cast polymethyl methacrylate, PMMA, which were ground and polished to achieve transparency. The operating conditions were derived from [13] where the original atomizer operated with JET A-1 at $p_{l}=0.5 \mathrm{MPa}$ roughly yield $R e=1000$. Other operating regimes, see Table 1 , covered $R e=2000$ and 4000 . This range of $R e$ values corresponds to the range of $p_{l}$ from 0.5 to $8 \mathrm{MPa}$ for the originally sized (10-times smaller) atomizer using JET A-1.

The operating liquid was p-cymene (1-Methyl-4-(propan-2-yl) benzene). It is one compound, colourless liquid, whose value of refractive index $n=1.49$ is closely matched to the atomizer body which simplifies the optical measurement and reduces the measurement errors. The physical properties of the p-cymene at room temperature are as follows: surface tension $\sigma=$ $0.028 \mathrm{~kg} / \mathrm{s}^{2}$, liquid dynamic viscosity $\mu_{l}=0.00085 \mathrm{~kg} /(\mathrm{m} \cdot \mathrm{s})$, and liquid density $\rho_{l}=848 \mathrm{~kg} / \mathrm{m}^{3}$. The identical test bench as in [12] was used. The uncertainty in pressure sensing was $0.05 \mathrm{kPa}$ and the calculated uncertainty of $C_{D}$ was $0.25 \%$ of actual value.

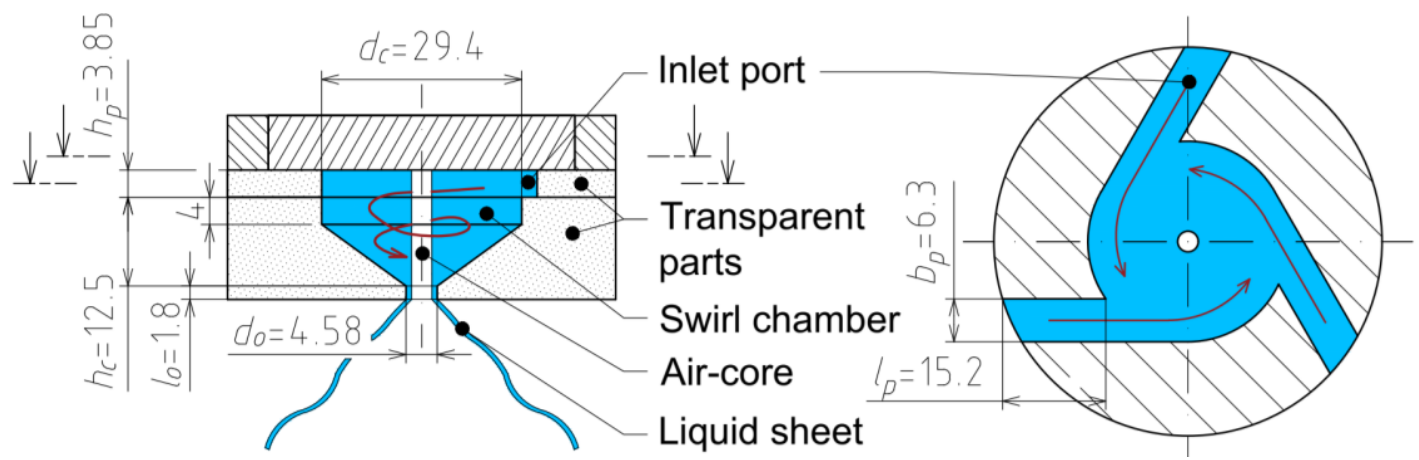

Figure 1 Atomizer schematic drawing with main dimensions in millimetres

Table 1 List of operating regimes

\begin{tabular}{llllllll}
\hline $\boldsymbol{R e}$ & $\boldsymbol{p}_{\boldsymbol{I}}$ & $\dot{\boldsymbol{m}}_{\boldsymbol{p}}$ & $\boldsymbol{v}_{\boldsymbol{p}}$ & $\boldsymbol{C}_{\boldsymbol{D}}$ & $\boldsymbol{F r}$ & $\boldsymbol{d}_{\text {ace }}$ & $\boldsymbol{d}_{\text {acc }}$ \\
\hline$[-]$ & {$[\mathrm{kPa}]$} & {$[\mathrm{kg} / \mathbf{h}]$} & {$[\mathbf{m} / \mathbf{s}]$} & {$[-]$} & {$[-]$} & {$[\mathbf{m m}]$} & {$[\mathbf{m m}]$} \\
\hline $\mathbf{1 0 0 0} \pm \mathbf{1 0}$ & 2.1 & 47.5 & 0.206 & 0.420 & 5.0 & 2.82 & 1.55 \\
\hline $\mathbf{2 0 0 0 \pm 1 0}$ & 8.8 & 94.8 & 0.411 & 0.410 & 9.9 & 2.99 & 1.64 \\
\hline $\mathbf{4 0 0 0 \pm \mathbf { 1 0 }}$ & 36.3 & 188.4 & 0.816 & 0.401 & 19.6 & 3.20 & 1.74 \\
\hline
\end{tabular}

\section{High-speed imaging}

A FASTCAM SA-Z high-speed camera (Photron, Japan) with long-distance microscope 12X Zoom lens (NAVITAR, New York, USA) which is composed of a 2X F-mount adapter (type 162922), a $12 \mathrm{~mm}$ F.F zoom lens (type 1-50486), and 0.25X lens (type 1-50011) was used to document the spatial and temporal behaviour of the air-core and discharged liquid sheet in one image with spatial dimensions of $31 \times 31 \mathrm{~mm}$. The camera frame rate was 20,000 frames per second, the resolution was $1024 \times 1024 \mathrm{px}$ and the shutter speed was set to $40 \mu \mathrm{s}$. A backlight illumination of the atomizer was provided using an LED panel. The air-core dimensions and the spray cone angle, SCA, were measured by an in-house MATLAB code. 

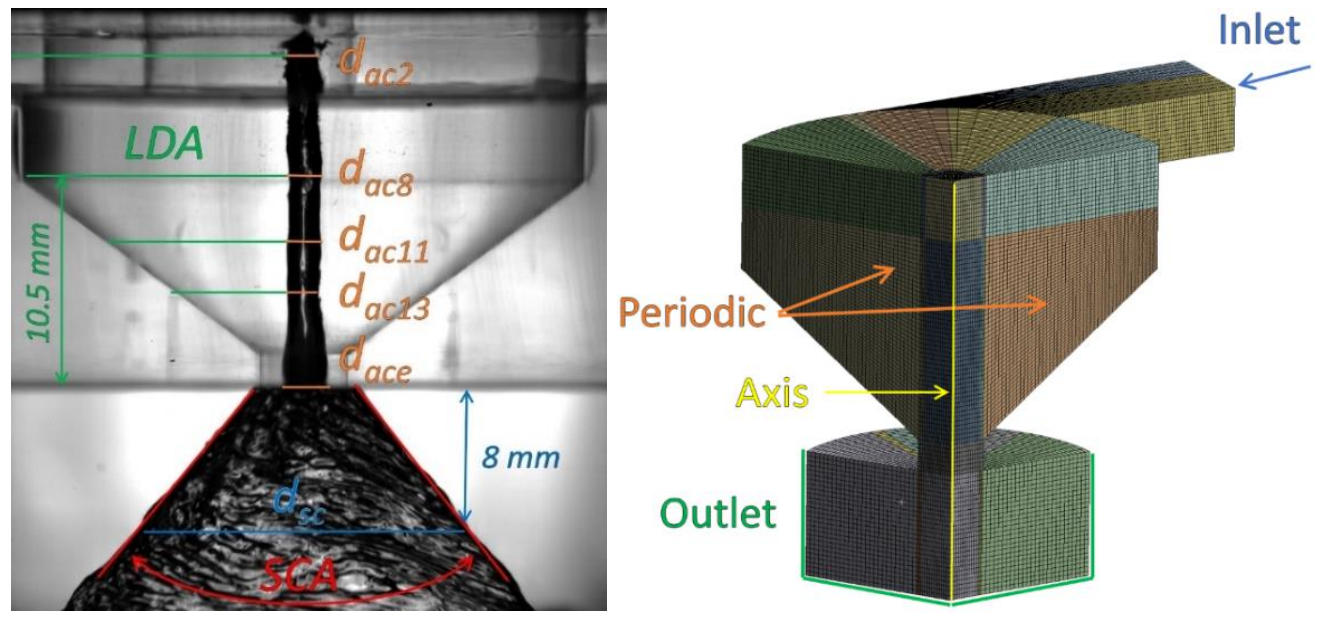

Figure 2 Left: The typical high-speed image with measured positions, Right: 3D periodic mesh with inlet port

\section{Numerical setup}

The CFD simulations were made using commercial software Ansys Fluent 19.2. The geometrical design of the atomizer allows for the use of periodic boundary condition, since the atomizer can be divided into three identical parts, each $120^{\circ}$ section. The structured mesh contained 686,300 cells, the mesh with prismatic boundary layer has the same structure but contains 6 prismatic cells in the wall boundary layer to reach the wall $y^{+} \sim 1$ along the exit orifice. The mesh independency test showed comparable results regardless the cell size (from $300 \mathrm{k}$ to $1 \mathrm{M}$ cells) and it is not discussed here for brevity. Pressure-velocity coupling was done using the PISO scheme for transient solution and the pseudo transient Coupled scheme for the steady solution. Turbulence and momentum used Second Order Upwind discretization. The liquid-air interaction was captured by a Volume of Fluid model with a geo-reconstruct scheme for transient models or Compressive scheme for the steady cases and LES. Surface tension between air and liquid was set as constant value. The air was treated with constant density. The gravity force was also considered. The transient solution used variable time stepping with a Courant number 0.15 . A typical time step size was approximately $2 \times 10^{-6} \mathrm{~s}$. After reaching a quasi-static solution, time averaging, with minimum of $0.1 \mathrm{~s}$, was applied.

Several turbulence models based on a Reynolds-averaged Navier-Stokes (RANS) equation, LES and laminar solver were considered. The mathematical description of the used methods and models is well known and can be found elsewhere [14]. Simple two-equation models represented by $k-\varepsilon$ and $k-\omega$ were chosen for their good accuracy and versatile use for industrial applications. These models determine a turbulent length scale and a time scale by solving two separate transport equations. The $k-\varepsilon$ model is based on a transport equation for kinetic energy $k$ and dissipation rate $\varepsilon$. In this paper, the RNG and realizable $k-\varepsilon$ models were used. The wall treatment was done using the scalable wall function for meshes without boundary layer and enhanced wall treatment (WT) was used for meshes with a prismatic boundary layer. The $k$ - $\omega$ SST model with low-Re correction was used with meshes with boundary layer only. This model combines the standard $k-\omega$ model for near wall treatment and the standard $k-\varepsilon$ model in the free stream flow. The Reynolds Stress model (RSM) is among the most advanced RANS models for the swirl dominant flows as it solves all the transport equations for the Reynolds stresses. This model was used with low-Re and shear flow correction, with scalable wall function for meshes with wall $y^{+}>10$ and omega-stress based for mesh with boundary layer. Large Eddy Simulation (LES) was represented by Wall-Adapting Local Eddy Viscosity model (WALE) with no inlet perturbations. For the turbulent models, the value of turbulence intensity of $1 \%$ and hydraulic diameter of $4.9 \mathrm{~mm}$ were set on the inlet. 


\section{Results and Discussion}

The results for both steady and transient ones are listed in Table 2 for $R e=2000$. The effect of $R e$ on the results from the selected models is shown in Figure 3. The both versions of steady $k-\varepsilon$, RNG and Realizable, performed almost identically and their results were close to the experimental data. The steady RSM reached the largest deviation from the experimental data, since it was unable to predict the air-core within the swirl chamber. This model also failed on the mesh with a prismatic layer using Enhanced wall treatment. The same model, but omegabased RSM, properly captured the air-core but underestimated the $C_{D}$. The similar results were obtained by the steady $k-\omega-S S T$ model. Both the $k-\omega-S S T$ and omega-based RSM predicted $C_{D}$ accurately in the transient simulation, where all the turbulence models return virtually identical results including LES. Only the omega-based RSM converged in the transient simulations, other variants of RSM diverged.

An improper setup of the wall function results in failure of the air-core prediction. The wall $y^{+}$ must be carefully checked before selecting the proper wall function as the standard wall function failed in the all investigated cases. The turbulence models were able to closely predict flow characteristics even for $R e=1000$, which is in good agreement with [8], where the $k-\varepsilon$ model returns reliable results for $R e=1600$. Note here that RSM model underperformed other turbulence models, similarly as in [8]. This result was not expected, since the RSM should be superior for flows with anisotropic turbulence, which is the case of swirl atomizer. It predicts the air-core only in the case of omega-based RSM, but its results were practically identical to the simpler $k-\omega$.

The suitable models from Table 2 were compared in the range of $R e$ and the results are presented in Figure 3. No RSM model was able to predict the air-core for $R e=1000$, thus these models are not presented here. The $C_{D}$ decreased in experiment slightly with $R e$ as $C_{D} \propto R e^{-0.035}$ or with $p_{l}$ as $C_{D} \propto p_{l}^{0.016}$. It is in good agreement with several authors $[15,16]$ who found the similar decrease in $C_{D}$. Nevertheless, all the CFD models predicted the same descending trend, yet minor differences were observed. The steady $k-\omega$ model underpredicted the $C_{D}$, particularly for $R e=1000$. The $k-\varepsilon$ also suffer from difficulties with this regime, where it severely overpredicts the $C_{D}$ and underpredicts the air-core dimension. The LES model returns the closest results of $C_{D}$, followed by the transient laminar model, which slightly diverts from the experimental data at $R e=4000$. Note here that the empirical correlation for $C_{D}$ proposed by Rizk and Lefebvre [17] gives a constant value of $C_{D}$ of 0.41 , which is in perfect agreement with the experimental data for $R e=2000$. The $C_{D}$ value is usually related to the air-core diameter. Therefore, a decrease in $C_{D}$ should be accompanied with the growing aircore diameter, which is true for both experimental data and all the CFD models, see Figure 3 , right. Only the $k-\varepsilon$ model diverts for $R e=1000$. Other models tend to slightly overestimate the air-core size for low $R e$.

The relative velocity of discharged liquid sheet, $v_{/} v_{p}$ (combined radial and axial velocity divided by the inlet velocity) is slightly increasing with $R e$. All CFD models captured the trend from the experiment well, but the velocity values were overestimated by most of them. The laminar model was closest to the experimental data for $R e=1000$ and 2000 , followed by the LES and $k-\varepsilon$. Slightly different results were obtained at the highest $R e$, where the LES outperformed the laminar model, but still overestimated the velocity by $5 \%$.

A huge disparity is found for the SCA results, where the value of experimental SCA is more than $20 \%$ larger than the predicted one. The experimental SCA discussed here is measured directly after discharge as the apex angle of cone, which covers the liquid sheet, while the SCA is based on the maximum in the liquid fraction inside the discharge liquid sheet. Nevertheless, both approaches should return very similar values. 
Table 2 Comparison of different physical models. 3D periodic mesh with port, $R e=2000$.

\begin{tabular}{|c|c|c|c|c|c|c|c|c|c|}
\hline Mesh & & Physical model & $p_{I}$ & SCA & $d_{a c e}$ & $\underset{m}{d_{a 11 m}}$ & $U_{I}$ & $V_{I}$ & $C_{D}$ \\
\hline & & & [kPa] & {$\left[{ }^{\circ}\right]$} & [mm] & [mm] & {$[\mathrm{m} / \mathrm{s}]$} & {$[\mathrm{m} / \mathrm{s}]$} & {$[-]$} \\
\hline Structured & \multirow{8}{*}{ 离 } & Laminar & 9.20 & 59.9 & 3.08 & 1.82 & 3.9 & 2.4 & 0.40 \\
\hline Structured & & $\begin{array}{l}\text { k- } \varepsilon \text { - Realizable } \\
\text { Scalable WF }\end{array}$ & 8.95 & 53.6 & 3.10 & 1.72 & 3.7 & 2.3 & 0.41 \\
\hline Structured & & $\begin{array}{c}\text { RSM } \\
\text { Scalable WF }\end{array}$ & 7.40 & 59.9 & 2.51 & 0 & 2.7 & 1.7 & 0.45 \\
\hline With prismatic layer & & $k-\omega-S S T$ & 10.4 & 62.0 & 3.10 & 1.56 & 4.1 & 2.9 & 0.38 \\
\hline With prismatic layer & & $\begin{array}{l}k-\varepsilon-\text { Realizable } \\
\text { Enhanced WT }\end{array}$ & 8.87 & 58.0 & 2.97 & 1.45 & 3.0 & 2.2 & 0.41 \\
\hline With prismatic layer & & $\begin{array}{c}k-\varepsilon-R N G \\
\text { Enhanced WT }\end{array}$ & 8.80 & 57.2 & 2.95 & 1.40 & 3.0 & 2.2 & 0.41 \\
\hline With prismatic layer & & RSM - Omega & 10.2 & 58.3 & 3.08 & 1.49 & 3.7 & 2.6 & 0.38 \\
\hline With prismatic layer & & $\begin{array}{c}\text { RSM } \\
\text { Enhanced WT }\end{array}$ & \multicolumn{7}{|c|}{ Diverged } \\
\hline Structured & \multirow{8}{*}{ 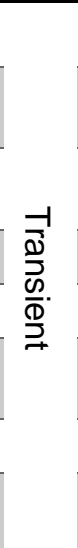 } & Laminar & 9.30 & 65.3 & 3.07 & 1.85 & 2.9 & 2.6 & 0.40 \\
\hline Structured & & $\begin{array}{l}\text { k- } \varepsilon \text { - Realizable } \\
\text { Scalable WF }\end{array}$ & 8.54 & 54.1 & 3.07 & 1.67 & 3.6 & 2.4 & 0.42 \\
\hline Structured & & $\begin{array}{c}\text { RSM } \\
\text { Scalable WF }\end{array}$ & \multicolumn{7}{|c|}{ Diverged } \\
\hline With prismatic layer & & LES - WALE & 8.96 & 59.9 & 3.03 & 1.55 & 3.7 & 2.6 & 0.41 \\
\hline With prismatic layer & & $k-\omega-S S T$ & 8.84 & 57.6 & 3.01 & 1.50 & 3.0 & 2.7 & 0.41 \\
\hline With prismatic layer & & $\begin{array}{l}k-\varepsilon-\text { Realizable } \\
\text { Enhanced WT }\end{array}$ & 8.05 & 52.0 & 2.87 & 1.22 & 3.1 & 2.3 & 0.43 \\
\hline With prismatic layer & & RSM - Omega & 9.10 & 54.0 & 3.02 & 1.56 & 3.1 & 2.6 & 0.41 \\
\hline With prismatic layer & & $\begin{array}{c}\text { RSM } \\
\text { Enhanced WT }\end{array}$ & \multicolumn{7}{|c|}{ Diverged } \\
\hline
\end{tabular}

The $S C A$ grows in the experiment with $R e$ as $S C A \propto R e^{0.12}$, respectively with $p_{l}$ as $S C A \propto p^{0.06}$, while it increases in transient laminar simulation as $S C A \propto R e^{0.06}$ or $S C A \propto p_{l}^{0.03}$ and it is constant for the LES simulation. The reason for these disparities is not clearly known, but it may be partially related to the flow of the surrounding air, which may slightly affect the liquid sheet formation as described in [18] and was not captured in CFD. Also, manufacturing inaccuracies and different measuring methods may introduce some errors. A widely used empirical correlation published by Rizk and Lefebvre [19] predicted much higher influence of the inlet pressure as $S C A \propto p_{l}^{0.11}$. However, this correlation was derived for liquids with higher viscosity and well captured the trends using higher viscous liquid in our previous study [12] using almost identical geometry as used here. The values of $S C A$ based on the Rizk predictions are 46, 54 and $63^{\circ}$ for $R e=1000,2000$ and 4000, respectively. These values are well below the measured values, but are much closer to the CFD prediction. Since the CFD predicts correctly other parameters of the internal flow, the differences in SCA might be linked with a different measurement technique, complex air-flow near the liquid sheet or wettability of the atomizer PMMA body.

\section{Conclusions}

The 3D numerical simulations of pressure-swirl atomizer internal flow were compared in transient and steady state with experimental data for $R e=1000,2000$ and 4000. Various numerical setups, including six turbulence models and several geometrical simplifications, were investigated. 

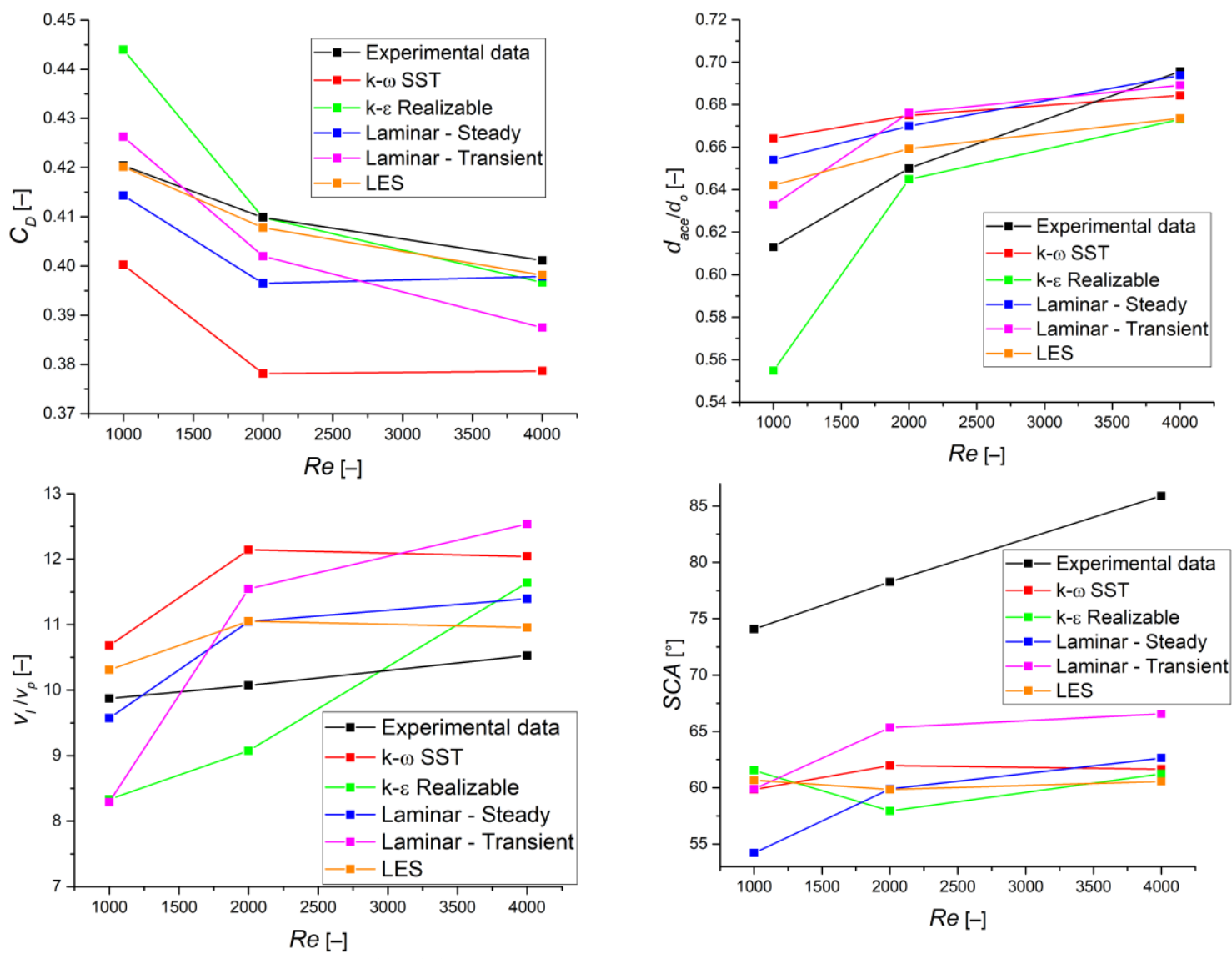

Figure 3 Effect of $R e$ on $C_{D}, S C A$, liquid sheet velocity, $v_{l}$, and $d_{a c e}$ from various numerical setups.

The steady simulations are comparable with the time-averaged transient ones; but their convergence rate is at least about an order of magnitude higher.

The laminar solver was capable to predict the $C_{D}$, air-core dimensions and velocity profiles with an error less than $5 \%$ compared with the experimental results in for the whole range of $R e$. The LES model performed similarly to the laminar solver for low $R e$ and was superior for $R e=4000$. The two-equation models, $k-\varepsilon$ and $k-\omega$, were sensitive to proper solving of the near wall flow and were not accurate for low Re. However, all the models captured well the trends. The worst results were surprisingly obtained for RSM (Reynolds Stress Model), which diverged for $R e=1000$ and 2000 or predicted the undeveloped air-core. For the unknown reason, the only parameter predicted with large error was the spray cone angle (SCA).

\section{Acknowledgments}

The authors acknowledge the financial support the project No. 18-15839S funded by the Czech Science Foundation and project "Computer Simulations for Effective Low-Emission Energy Engineering" funded as project No. CZ.02.1.01/0.0/0.0/16_026/0008392 by Operational Programme Research, Development and Education, Priority axis 1: Strengthening capacity for high-quality research and project LTAIN19044 funded from the INTER EXCELLENCE program by the Czech Ministry of Education, Youth and Sports.

\section{Nomenclature}

$\begin{array}{llll}C_{D} & \text { discharge coefficient }[-] & \mu_{l} & \text { liquid dynamic viscosity }[\mathrm{kg} /(\mathrm{m} \cdot \mathrm{s})] \\ d_{a c e} & \text { air-core diameter in exit orifice }[\mathrm{mm}] & p_{l} & \text { pressure drop at the nozzle }[\mathrm{Pa}]\end{array}$ 


$\begin{array}{llll}d_{a 11} & \text { air-core diameter in } 11 \mathrm{~mm} \text { from top } & R e & \text { Reynolds number inside inlet po } \\ \text { of the swirl chamber }[\mathrm{mm}] & & \\ d_{0} & \text { exit orifice diameter }[\mathrm{mm}] & S C A & \left.\text { spray cone angle }{ }^{\circ}\right] \\ F r & \text { Froude number }[-] & U_{l} & \text { axial velocity }[\mathrm{m} / \mathrm{s}] \\ \dot{m} & \text { mass flow rate }[\mathrm{kg} / \mathrm{h}] & v_{p} & \text { mean velocity in inlet port }[\mathrm{m} / \mathrm{s}] \\ n & \text { refractive index } & V_{l} & \text { radial velocity }[\mathrm{m} / \mathrm{s}]\end{array}$

\section{References}

[1] Maly, M., Jedelsky, J., Slama, J., Janackova, L., Sapik, M., Wigley, G., and Jicha, M., 2018, "Internal flow and air core dynamics in Simplex and Spill-return pressure-swirl atomizers," International Journal of Heat and Mass Transfer, 123, pp. 805-814.

[2] Amini, G., 2016, "Liquid flow in a simplex swirl nozzle," International Journal of Multiphase Flow, 79, pp. 225-235.

[3] Wimmer, E., and Brenn, G., 2013, "Viscous flow through the swirl chamber of a pressure-swirl atomizer," International Journal of Multiphase Flow.

[4] Yule, A., and Chinn, J., 1997, "Pressure swirl atomizer internal flow and performance," ILASS, Americas, pp. 205-209.

[5] Yule, A. J., and Chinn, J. J., 2000, "The internal flow and exit conditions of pressure swirl atomizers," Atomization and Sprays, 10(2), pp. 121-146.

[6] Chinn, J. J., 2008, "The numerics of the swirl atomizer," ILASS, p. 7.

[7] Madsen, J., Hjertager, B. H., and Solberg, T., 2004, "Numerical simulation of internal flow in a largescale pressure-swirl atomizer," ILASS, pp. 183-188.

[8] C. Galbiati, S. Tonini, P. Conti, and Cossali, G. E., "Numerical Simulations of Internal Flow in an Aircraft Engine Pressure Swirl Atomizer," Journal of Propulsion and Power(6), p. 1433.

[9] Qian, W., Hui, X., Zhang, C., Xu, Q., Lin, Y., and Sung, C.-J., "A numerical study of the internal flow in a pressure swirl atomizer," Proc. ASME Turbo Expo 2017: Turbomachinery Technical Conference and Exposition, American Society of Mechanical Engineers Digital Collection.

[10] Nouri-Borujerdi, A., and Kebriaee, A., 2012, "Numerical simulation of laminar and turbulent twophase flow in pressure-swirl atomizers," AIAA journal, 50(10), pp. 2091-2101.

[11] Abbasi Baharanchi, A., Nordin Darus, A., Ansari, M., and Abbasi Baharanchi, E., 2012, "An Optimum Method of Capturing Interface and a Threshold Weber Number for Inclusion of Surface Tension Force in Simulation of Nozzle Internal Flow in Pressure Swirl Atomizers," (ASME International Mechanical Engineering Congress and Exposition), p. 12.

[12] Maly, M., Cejpek, O., Sapik, M., Ondracek, V., Wigley, G., and Jedelsky, J., 2021, "Internal flow dynamics of spill-return pressure-swirl atomizers," 120, p. 110210.

[13] Maly, M., Sapik, M., Cejpek, O., Wigley, G., Katolicky, J., and Jedelsky, J., 2019, "Effect of spill orifice geometry on spray and control characteristics of spill-return pressure-swirl atomizers," Experimental Thermal and Fluid Science, 106, p. 12.

[14] Ansys ${ }^{\circledR}, 2017$, Fluent Theory Guide, Release 18.2 .

[15] Halder, M., Dash, S., and Som, S., 2004, "A numerical and experimental investigation on the coefficients of discharge and the spray cone angle of a solid cone swirl nozzle," Experimental thermal and fluid science, 28(4), pp. 297-305.

[16] Som, S. K., 1983, "Theoretical and experimental studies on the coefficient of discharge and spray cone angle of a swirl spray pressure nozzle using a power-law non-Newtonian fluid," 12(1), pp. 39-68.

[17] Rizk, N. K., and Lefebvre, A. H., 1985, "Internal flow characteristics of simplex swirl atomizers," Journal of Propulsion and Power, 1(3), pp. 193-199.

[18] Jedelsky, J., Maly, M., del Corral, N. P., Wigley, G., Janackova, L., and Jicha, M., 2018, "Air-liquid interactions in a pressure-swirl spray," International Journal of Heat and Mass Transfer, 121, pp. 788804.

[19] Rizk, N. K., and Lefebvre, A. H., 1987, "Prediction of velocity coefficient and spray cone angle for simplex swirl atomizers," International Journal of Turbo and Jet Engines, 4(1-2), pp. 65-74.

[20] Ballester, J., and Dopazo, C., 1994, "Discharge coefficient and spray angle measurements for small pressure-swirl nozzles," Atomization and sprays, 4(3). 